\title{
A first-in-Asian phase 1 study to evaluate safety, pharmacokinetics and clinical activity of VS-6063, a focal adhesion kinase (FAK) inhibitor in Japanese patients with advanced solid tumors
}

\author{
Toshio Shimizu $^{1} \cdot$ Kazuya Fukuoka $^{1} \cdot$ Masayuki Takeda $^{1}$ Tutomu Iwasa ${ }^{1}$. \\ Takeshi Yoshida $^{1} \cdot$ Joanna Horobin $^{2} \cdot$ Mitchell Keegan $^{2} \cdot$ Lou Vaickus $^{2}$ - Ajit Chavan ${ }^{2}$. \\ Mahesh Padval $^{2} \cdot$ Kazuhiko Nakagawa $^{1}$
}

Received: 23 February 2016 / Accepted: 14 March 2016 / Published online: 30 March 2016

(C) The Author(s) 2016. This article is published with open access at Springerlink.com

\begin{abstract}
Purpose VS-6063 (also known as defactinib or PF-04554878) is a second-generation inhibitor of focal adhesion kinase and proline-rich tyrosine kinase-2. This phase 1 study evaluated the safety and tolerability, pharmacokinetics, and clinical activity of VS-6063 in Japanese subjects with advanced solid tumor malignancies in a firstin-Asian study setting.

Methods VS-6063 was administered orally twice daily (b.i.d.) in 21-day cycles to cohorts of three subjects each with a standard $3+3$ dose-escalation design until disease progression or unacceptable toxicity. Blood samples for pharmacokinetics were collected on Day 1 and 15 . The assessments were performed using CTCAE v4.0 for adverse events (AEs), and the Response Evaluation Criteria In Solid Tumors, version v1.1 (RECIST v1.1) for tumor response.

Results Nine patients were treated across three dose levels (200-600 mg BID). No dose-limiting toxicities were observed at any dose level. Most frequent treatment-related AEs were Grade 1/2 unconjugated hyperbilirubinemia, fatigue, decreased appetite, and diarrhea. Only one subject in the $200 \mathrm{mg}$ BID cohort experienced reversible and transient Grade 3 unconjugated hyperbilirubinemia. PK
\end{abstract}

Presented at the 26th EORTC-NCI-AACR Symposium on "Molecular Targets and Cancer Therapeutics", held in Barcelona, Spain, November 18-21, 2014.

Toshio Shimizu

jcog9511@ @otmail.co.jp

1 Department of Medical Oncology, Kindai University Faculty of Medicine, 377-2 Ohno-higashi, Osaka-Sayama City, Osaka 5898511, Japan

2 Verastem, Inc., Needham, MA 02494, USA analyses confirmed that the exposure at the recommended Phase 2 dose (RP2D) of $400 \mathrm{mg}$ BID was comparable with exposures previously reported in non-Japanese subjects. Durable stable disease of approximately 24 weeks was confirmed in two subjects (malignant mesothelioma and rectal cancer).

Conclusions VS-6063 was well tolerated at all dose levels investigated in this first-in-Asian study. These data support the administration of VS-6063 to Japanese subjects at the RP2D in clinical trials involving solid tumor malignancies.

Keywords Focal adhesion kinase $\cdot$ Proline-rich tyrosine kinase-2 · First-in-Asian phase 1 study · VS-6063 · Defactinib

\section{Introduction}

High expression of focal adhesion kinase (FAK) has been frequently demonstrated to be associated with invasive and metastatic malignancies, implicating FAK in malignant progression of multiple epithelial tumors [1-6]. FAK localizes to focal adhesions and mediates physical attachment of cells to the extracellular matrix (ECM). Focal adhesion kinase is known to promote tumor cell survival and resistance to anoikis (induction of apoptosis upon loss of contact with the ECM), via the ADRB2/Src signaling pathway. The ability to survive without ECM contact is a hallmark of metastatic cells, allowing them to leave the parent tumor and migrate to and colonize distant tissues. This ability is also characteristic of a subpopulation of highly tumorigenic cells which possess qualities like stem cell. These cells are considered to be cancer stem cells (CSC) or cancer or tumor initiating cells (CIC or TIC) and may be defined as malignant cells with a stem cell phenotype [7]. Tumor 
growth thus occurs by propagation of these cells, and CSC have been implicated in tumor initiation, progression, metastasis, and drug resistance. This mechanism of metastatic dissemination has recently been shown to involve the formation of filopodium-like protrusions and the subsequent establishment of elongated, mature adhesion plaques which contribute critically to the rapid proliferation of the micrometastatic cells within the new environment [8]. In these studies, activation of FAK was shown to be a critical protein involved in the proliferation of these cells. Taken together these data suggest that FAK modulation may be a potential therapeutic target for cancer.

VS-6063 (defactinib) is a small molecule, orally available potent adenosine $5^{\prime}$-triphosphate (ATP)-competitive, reversible inhibitor of FAK and proline-rich tyrosine kinase-2 (Pyk2). FAK and Pyk2 are members of the same family of nonreceptor protein tyrosine kinases sharing significant sequence homology and are implicated as important integrating molecules in signal transduction cascades. VS-6063 produces potent in vitro inhibition of recombinant human FAK and Pyk2 activity (inhibitory concentration of $50 \%$ [IC50] $=0.6 \mathrm{nM}$ for each kinase), displaying more than 100-fold greater selectivity for FAK and Pyk2 than for other nontarget kinases. Toxicology studies conducted with VS-6063 have identified the liver and gall bladder, gastrointestinal tract, hematopoietic system, testes/ epididymis, and cardiovascular system as potential targets. Importantly, preclinical evidence indicates that VS-6063 has a low potential for CYP 2C-, 2D- or 3A-mediated drug interactions. Based on both these preclinical findings and previous first-in-human (FIH) experience of phase 1 study in subjects with advanced solid tumors in USA [9], a firstin-Asian phase 1 dose-escalation study was conducted to determine the MTD and overall safety and tolerability of VS-6063, and to identify a recommended phase 2 dose (RP2D) in Japanese patients with non-hematologic malignancies. Secondary objectives included characterization of the pharmacokinetic profile and documentation of preliminary antitumor activity.

\section{Materials and methods}

\section{Patient eligibility}

All subjects provided informed consent, and the study was conducted in accordance with both the Declaration of Helsinki and Good Clinical Practices (GCP), including the archiving of essential documents. The study was approved by the institutional review board of study site, and this study was registered at ClinicalTrials.gov as NCT01943292. The main eligibility criteria were as follows: Japanese patient with histologically or cytologically confirmed diagnosis of locally advanced or metastatic solid tumor malignancies that had experienced disease progression on, were intolerant of, or not eligible for standard therapy; aged 20 years or older; an Eastern Cooperative Oncology Group (ECOG) performance status of 0 or 1 ; and adequate hematologic, hepatic, and renal functions. Key exclusion criteria included cancer-directed therapy (chemotherapy, radiotherapy, hormonal therapy, biologic, or immunotherapy, etc.) within 28 days of the first dose of study drug or 5 half-lives, whichever was shorter, a gastrointestinal condition which could have interfered with the swallowing or absorption of study medication, corrected QT interval $\left(\mathrm{QT}_{\mathrm{c}}\right) \geq 470 \mathrm{~ms}$ (as calculated by the Fridericia correction formula), uncontrolled or severe concurrent medical condition (including uncontrolled brain metastases), and uncontrolled or severe cardiovascular disease. Potent CYP3A4 inhibitors or inducers and systemic anticoagulation were prohibited.

\section{Study design and treatment}

This was a phase 1, open-label, dose-escalation study to evaluate the safety and PK of VS-6063, a FAK inhibitor in Japanese subjects with advanced solid tumor malignancies. This study was expected to enroll up to 18 evaluable subjects with non-hematologic malignancies if 6 subjects were assigned at each of the 3 planned dose levels of 200, 400, and $600 \mathrm{mg}$ BID. VS-6063 was administered orally, BID, in continuous 21 day cycles. Subject enrollment proceeded according to a standard $3+3$ design. In the absence of DLT, each subject received oral VS-6063 (BID) for a minimum of 21 days of continuous daily dosing (1 cycle) and could continue to receive additional cycles of study treatment until disease progression was documented or other treatment discontinuation criteria were met. All patients within a cohort were required to complete 1 cycle ( 21 days) of dosing with $<1$ of 3 or $<2$ of 6 subjects experiencing a DLT prior to escalation to the next dose level in a new cohort. Three subjects were to be treated at each dose level until the first instance of DLT, after which up to 6 subjects were to be treated at that dose level. If a second DLT was observed in up to 6 subjects, the DLT dose level was reached. DLTs included Grade 4 neutropenia lasting $\geq 7$ days without growth factor support; Grade $\geq 3$ febrile neutropenia; Grade 4 thrombocytopenia, Grade 3 or 4 nonhematologic toxicities (excluding nausea, vomiting, or diarrhea unless uncontrolled with supportive care), Grade $\geq 3 \mathrm{QT}_{\mathrm{c}}$ interval prolongation, or any treatment-related toxicities that resulted in failure to receive at least approximately $85 \%$ of the planned doses for that cycle (e.g., failure to complete at least 18 days of treatment in a continuous 21-day regimen) despite maximal (as judged by the investigator) supportive care 
measures. The MTD was defined as the highest dose level studied at which $<1$ subject out of 3 or $<2$ subjects out of 6 experienced a DLT. The RP2D was to be determined in discussion among the sponsor, medical monitor, and investigators. Observations related to PK and any VS6063-related toxicities were included in the rationale supporting the RP2D. Safety and tolerability was assessed by the incidence and severity of AEs as determined by National Cancer Institute (NCI) Common Terminology Criteria for Adverse Events (CTCAE v4.03). The antitumor activity of study treatment was assessed according to the Response Evaluation Criteria in Solid Tumors (RECIST v1.1).

\section{Pharmacokinetic assessments}

\section{Blood sampling and processing}

Pharmacokinetic samples were to be collected for determination of VS-6063 concentration and potential metabolites at the time points of Pre-dose (within $30 \mathrm{~min}$ of VS-6063 dosing), postdose (15 min, $30 \mathrm{~min}$, and 1, 2, 4, 8, 12, and $24 \mathrm{~h}$ after VS-6063 administration) during Cycle 1 on Days 1 and 15. The analytical laboratory measured plasma concentrations using a validated method. Phoenix WinNonlin (v 6.3, Pharsight Inc., Mountain View, CA) was used to estimate all pharmacokinetic parameters using the non-compartmental analysis tool and the plasma model. $C_{\max }$ and $T_{\max }$ were determined by direct assessment (no calculations) of the concentration versus time data. All AUC calculations followed the linear trapezoidal rule. As data permitted, the terminal elimination rate constant (lambda $z, \lambda z$ ) for VS-6063 data was calculated. Lambda $z$ was determined by the slope of the regression line of the natural $\log$ transformed concentrations versus time with at least three data points not including the $C_{\max }$ and a correlation coefficient $\left(R^{2}\right)$ of regression $>0.90$. In instances where the $\lambda z$ range was not at least twofold greater than the calculated half-life, the resulting values (AUCINF, CL/F and $V_{\mathrm{z}} / F$ ) were deemed unreliable and were not reported, although the half-life will be calculated and reported if the $\lambda z$ range met the $R^{2}$ requirements. The following PK parameters were to be calculated: oral clearance $(\mathrm{CL} / F)$, maximum concentration $\left(C_{\max }\right), T_{\max }$, half-life (T1/2), area under the curve (AUC), and other relevant parameters.

\section{Urine collection and sampling}

To assess the elimination of VS-6063 and its potential metabolites, total 24-h urine output was to be collected on Cycle 1 Day 1 and Cycle 1 Day 15 in conjunction with PK sampling.

\section{Results}

\section{Patient characteristics}

A total of 9 subjects received VS-6063 between September 2013 and June 2014. All were Asian (of Japanese descent). The study population was predominantly male $(77 \%)$, with an ECOG performance status of 0 or $1(100 \%)$. The most common cancer type was rectal cancer (3 patients). Other cancer types, including esophageal, lung, mesothelioma, Paget's carcinoma, colon, and thymic cancer, were each reported in 1 subject. Eight subjects overall had received prior systemic therapy for their cancer, with a median of 7 prior therapies across dose cohorts. Six subjects, 2 in each dose cohort, had prior surgery. Only 1 subject, in the $200 \mathrm{mg}$ cohort, had received prior radiation therapy (Table 1).

\section{Safety and tolerability}

Treatment-related adverse events (AEs) occurring in at least two subjects are summarized in Table 2. The most commonly reported AEs overall were unconjugated hyperbilirubinemia (7 patients, $78 \%$ ), fatigue (6 patients, $67 \%$ ), decreased appetite (4 patients, $44 \%$ ), and diarrhea (3 patients, $33 \%$ ). Only one patient in the $200 \mathrm{mg}$ dose cohort experienced a Grade $3 \mathrm{AE}$ of unconjugated hyperbilirubinemia. All other toxicities were manageable and were predominantly mild in intensity (Grade 1 or Grade 2) in severity.

Table 1 Baseline characteristics of the patients $(n=9)$

\begin{tabular}{ll}
\hline Characteristics & All patients, $n=9$ \\
\hline Age (years) & 60.4 \\
Median & $38.0-75.0$ \\
Range & \\
Sex & 7 \\
Male & 2 \\
Female & \\
ECOG performance status & 7 \\
0 & 2 \\
1 & \\
Primary tumor & 4 \\
Colorectal cancer & 1 \\
Esophageal cancer & 1 \\
Mesothelioma & 1 \\
NSCLC & 1 \\
Thymic cancer & 1 \\
Extramammary Paget's disease & \\
No. of prior systemic therapy & 7 \\
Median & $0-13$ \\
Range &
\end{tabular}

ECOG Eastern Cooperative Oncology Group 
Table 2 Treatment-related adverse events occurring in at least 2 subjects

\begin{tabular}{|c|c|c|c|c|}
\hline & \multicolumn{3}{|c|}{ VS-6063 dose cohort } & \multirow{2}{*}{$\begin{array}{l}\text { Total } \\
N=9 \\
n(\%)\end{array}$} \\
\hline & $\begin{array}{l}200 \mathrm{mg} \text { BID } \\
N=3 \\
n(\%)\end{array}$ & $\begin{array}{l}400 \mathrm{mg} \text { BID } \\
N=3 \\
n(\%)\end{array}$ & $\begin{array}{l}600 \mathrm{mg} \text { BID } \\
N=3 \\
n(\%)\end{array}$ & \\
\hline Blood bilirubin increased & $3(100.0)$ & $2(66.7)$ & $2(66.7)$ & $7(77.8)$ \\
\hline Fatigue & $2(66.7)$ & $1(33.3)$ & $3(100.0)$ & $6(66.7)$ \\
\hline Decreased appetite & $2(66.7)$ & $1(33.3)$ & $1(33.3)$ & $4(44.4)$ \\
\hline Diarrhea & 0 & $1(33.3)$ & $2(66.7)$ & $3(33.3)$ \\
\hline Aspartate aminotransferase increased & $1(33.3)$ & 0 & $1(33.3)$ & $2(22.2)$ \\
\hline Blood alkaline phosphatase increased & $2(66.7)$ & 0 & 0 & $2(22.2)$ \\
\hline Nausea & $1(33.3)$ & 0 & $1(33.3)$ & $2(22.2)$ \\
\hline Headache & 0 & $1(33.3)$ & $1(33.3)$ & $2(22.2)$ \\
\hline Anemia & 0 & 0 & $2(66.7)$ & $2(22.2)$ \\
\hline
\end{tabular}

There were no AEs leading to death or SAEs, and no AEs leading to early study withdrawal. No DLTs were reported in any dose cohort. Hyperbilirubinemia was asymptomatic and its onset typically occurred within the first 2 weeks of initiating treatment. Patients with Grade 1 or 2 unconjugated hyperbilirubinemia were able to continue dosing, although bilirubin levels tended to fluctuate during treatment. Hyperbilirubinemia was reported across all dose cohorts, for $3(100 \%)$ patients in the $200 \mathrm{mg}$ dose cohort, $2(67 \%)$ patients in the $400 \mathrm{mg}$ dose cohort, and $2(67 \%)$ patients in the $600 \mathrm{mg}$ dose cohort. One event of hyperbilirubinemia (200 mg cohort) was Grade 3 in severity. This patient had Grade 1-2 hyperbilirubinemia starting on Day 7; the Grade 3 event began on Day 42 and resolved 6 days after onset following interruption of study drug. All reports of hyperbilirubinemia were considered to be related to defactinib. None of these subjects had concurrent increases in ALT or AST above ULN. The most common events of gastrointestinal disorders were diarrhea reported in 3 (33\%) subjects and nausea reported in $2(22 \%)$. Diarrhea was reported in $1(33 \%)$ subject in the $400 \mathrm{mg}$ dose cohort and $2(67 \%)$ subjects in the $600 \mathrm{mg}$ dose cohort. Nausea was reported in $1(33 \%)$ subject in the $200 \mathrm{mg}$ dose cohort, and $1(33 \%)$ subject in the $600 \mathrm{mg}$ dose cohort. Both reports of nausea were mild in severity as were 2 of the 3 reports of diarrhea; 1 subject in the $600 \mathrm{mg}$ group experienced diarrhea of moderate intensity. No clinically meaningful changes in any ECG parameter were observed for any dose cohort and no subject had a $\mathrm{QT}_{\mathrm{c}}$ interval $\geq 500 \mathrm{~ms}$ or $\mathrm{QT}_{\mathrm{c}}$ increase from baseline $>30 \mathrm{~ms}$.

\section{Pharmacokinetics}

VS-6063 was rapidly absorbed, with median $T_{\max }$ observed at $2.0 \mathrm{~h}$ (range $0.5-4.0 \mathrm{~h}$ ) postdose following oral doses of 200-600 mg BID. Plasma VS-6063 exposure $\left(C_{\max }\right.$ and AUC) increased in a less than dose proportional manner and the mean AUC0-12 and AUC0-24 values remained relatively consistent across the full dose range evaluated (Fig. 1). Doses above $400 \mathrm{mg}$ BID did not result in a significant increase in VS-6063 exposure. A similar $C_{\max }$ was observed in the 400 and $600 \mathrm{mg}$ BID dose cohorts on both Days 1 and 15 and mean AUC values were relatively consistent across the 200-600 mg BID dose range. Clearance appeared to be increased with dose on both Days 1 and 15, which is consistent with the less than dose proportional increase in exposure. Median half-life values ranged from 2.3 to $4.3 \mathrm{~h}$ across all dose regimens and both PK study days. The Day 1 mean CL/F values were 45.6, 105, and $204 \mathrm{~L} / \mathrm{h}$ for the 200, 400, and $600 \mathrm{mg}$ doses, respectively. The Day 15 mean CL/F values were 32. 1, 70, and $123 \mathrm{~L} / \mathrm{h}$ for the 200, 400, and $600 \mathrm{mg}$ doses, respectively (Table 3). VS-6063 was detected in the urine of all patients and appeared consistent across all dose cohorts. The mean renal clearance (CLr) values ranged from 0.0855 to $0.179 \mathrm{~L} / \mathrm{h}$, and the percent relative to the total dose administered ranged from 0.0439 to $0.356 \%$. All 9 subjects had systemic concentrations of the 4 metabolites of VS-6063 that were evaluated (M2, M3, M4, and M5). Median plasma $T_{\max }$ values for all metabolites were observed at 2.0-4.0 $\mathrm{h}$ postdose administration for all cohorts on both PK sampling days. Based on the relative plasma $C_{\max }$ and AUC0-12 values for the metabolites compared to VS-6063 values, M2 was the most abundant metabolite, followed by M4, M3, and then M5. Both the M2 and M4 exposures appeared to be $>10 \%$ of the parent exposure, while M3 and M5 had exposures that were $<10 \%$ of the parent exposure. In the urine, the M2 metabolite was the most abundant and was excreted in amounts greater than the parent compound.

\section{Antitumor activity}

The best response overall was stable disease for 3 (33\%) out of 9 subjects, 1 subject in each dose cohort. 


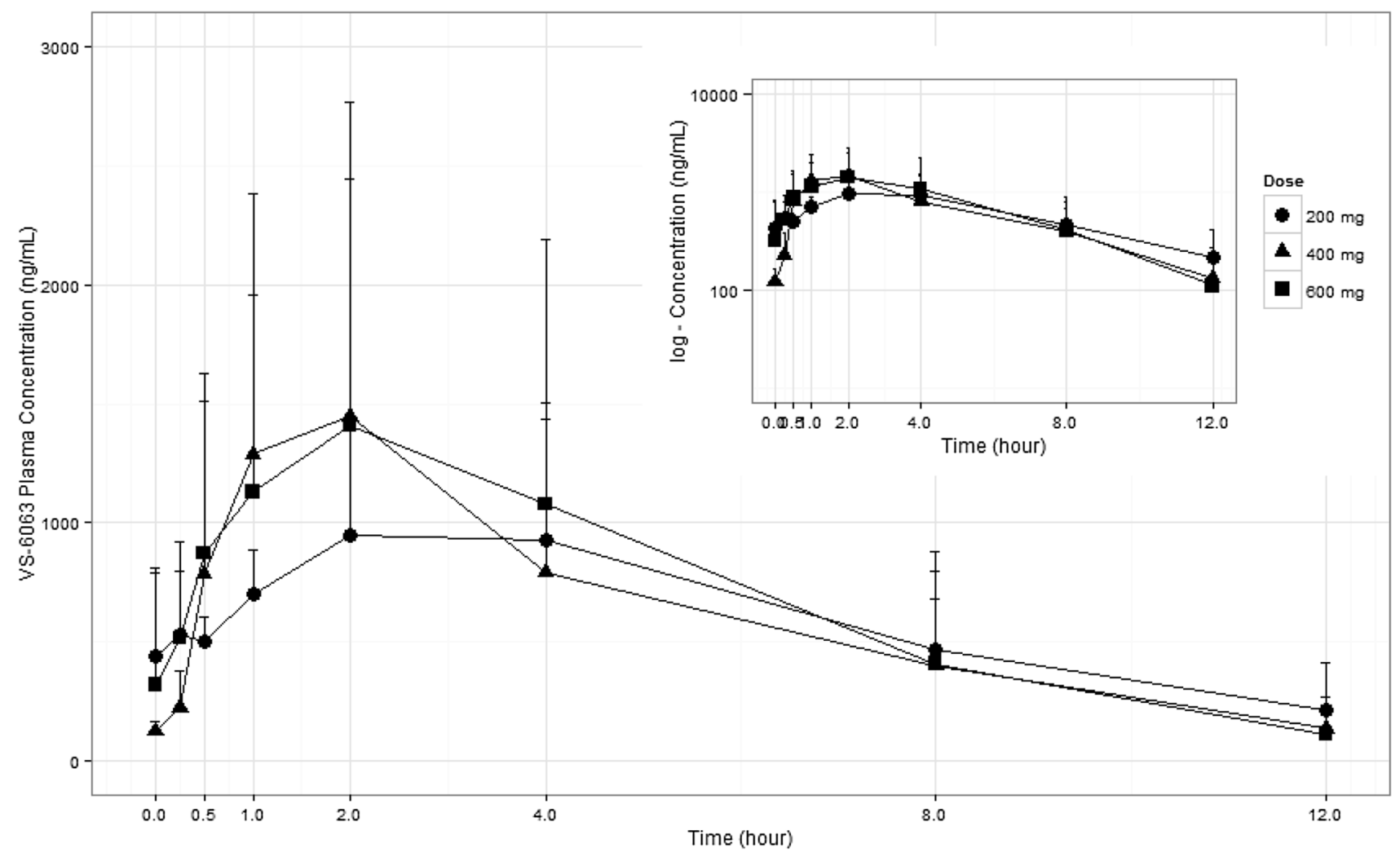

Fig. 1 Steady-state serum VS-6063 concentration-time profiles over the 12-h dose interval (VS-6063 doses 200-600 mg twice daily). PK analysis of previous first-in-human phase 1 study revealed that VS-6063 dose of $163 \mathrm{mg}$ twice daily (per 70-kg person) is projected to achieve a steady-state average plasma concentration equivalent to the predicted efficacious free (non-protein-bound) plasma concentration of $13.3 \mathrm{ng} / \mathrm{mL}$ required to produce $50 \%$ inhibition of tumor pFAK activity in humans

Table 3 Serum pharmacokinetic parameters of VS-6063 in Japanese patients with advanced solid tumors on Day 1 and Day 15

\begin{tabular}{|c|c|c|c|c|c|c|c|c|}
\hline Dose & Day & $\begin{array}{l}C_{\max }, \mu \mathrm{g} / \mathrm{mL} \\
\mathrm{GM}(\mathrm{CV} \%)\end{array}$ & $\begin{array}{l}T_{\max }, \mathrm{h}, \text { median } \\
\text { (range) }\end{array}$ & $\begin{array}{l}\mathrm{AUC}_{0-12 \mathrm{~h}}, \mu \mathrm{g} \mathrm{h} / \mathrm{mL} \\
\mathrm{GM}(\mathrm{CV} \%)\end{array}$ & $\begin{array}{l}\mathrm{AUC}_{0-\infty}, \mu \mathrm{g} \mathrm{h} / \mathrm{mL} \\
\mathrm{GM}(\mathrm{CV} \%)\end{array}$ & $\begin{array}{l}t_{1 / 2}, \mathrm{~h}, \text { median } \\
\text { (range) }\end{array}$ & $\begin{array}{l}V_{\mathrm{z}}, \mathrm{L} \\
\mathrm{GM}(\mathrm{CV} \%)\end{array}$ & $\begin{array}{l}\mathrm{CL} / \mathrm{F}(\mathrm{L} / \mathrm{h}) \\
\mathrm{GM}(\mathrm{CV} \%)\end{array}$ \\
\hline \multirow{2}{*}{$\begin{array}{l}200 \mathrm{mg} \text { BID } \\
n=3\end{array}$} & Day 1 & 11) & & $3.78(153)$ & $4.39(175)$ & $4.3(3.5,5.5)$ & $288(144)$ & $45.6(175)$ \\
\hline & Day 15 & $0.93(55)$ & $2(1,4)$ & $6.24(90)$ & NA & $2.95(2.9,3.0)$ & $334,99.6^{*}$ & $32.1(90)$ \\
\hline \multirow{2}{*}{$\begin{array}{l}400 \mathrm{mg} \text { BID } \\
n=3\end{array}$} & Day 1 & $0.67(333)$ & $2(2,4)$ & 3.37 (159) & $3.84(131)$ & $2.45(2.3,3.5)$ & 410 (189) & $105(133)$ \\
\hline & Day 15 & $0.96(218)$ & $2(1,4)$ & $5.71(136)$ & NA & $3.0(2.89,3.11)$ & $174(63.5)$ & 70 (136) \\
\hline \multirow{2}{*}{$\begin{array}{l}600 \mathrm{mg} \text { BID } \\
n=3\end{array}$} & Day 1 & $0.61(319)$ & $2(1,2)$ & $2.68(269)$ & $3.0(232)$ & $2.79(1.33,5.24)$ & $790(576)$ & $204(242)$ \\
\hline & Day 15 & $0.98(216)$ & $2(2,4)$ & $4.88(322)$ & NA & $2.27(1.58,2.95)$ & $158,3060^{*}$ & $123(322)$ \\
\hline
\end{tabular}

$\mathrm{AUC}_{0-12 \mathrm{~h}}$, area under the concentration-time curve from time zero to $12 \mathrm{~h}$ (dosing interval); $\mathrm{AUC}_{0-\infty}$, are under the concentration-time curve from time zero to infinity; $C_{\max }$, maximum observed serum concentration; $\mathrm{CV}$, coefficient of variation; PK, pharmacokinetic; $t 1 / 2$ elimination half-life; $T_{\max }$, time of maximum observed serum concentration; GM, Geometric mean

$* N=2$; therefore, individual values are presented

Remarkably, durable stable diseases by RECIST criteria were maintained for 169 days ( 24 weeks) in a subject with mesothelioma in the $400 \mathrm{mg}$ cohort, and for 162 days (23 weeks) in a subject with rectal cancer in the $600 \mathrm{mg}$ dose cohort. The overall median time to disease progression for all subjects was 63 days (9 weeks) and ranged from 61 to 64 days across dose cohorts.

\section{Discussion}

This was a first-in-Asian phase 1, open-label, dose-escalation study to evaluate the safety and PK of VS-6063 in subjects of Japanese descent with non-hematologic malignancies. The overall purpose of this phase 1 study was to support the development of VS-6063 as an oral 
formulation for the treatment of Asian (Japanese) subjects with advanced solid tumors. The doses and regimen proposed for this study were based on both nonclinical data and findings of the FIH phase 1 study. The rationale for a BID schedule was based on nonclinical data supporting the requirement for prolonged suppression of the target for maximal efficacy and the observed human half-life of $9.0 \mathrm{~h}$. In the previous FIH phase 1 study, subjects received doses of 12.5-750 mg of defactinib BID for continuous 21 day cycles. PK exposure of VS-6063 at the RP2D of $400 \mathrm{mg}$ BID was consistent with previously reported data in nonJapanese patients. Doses above $400 \mathrm{mg}$ BID did not result in a significant increase in VS-6063 exposure. Available data indicate that $400 \mathrm{mg}$ BID should result in adequate plasma concentration levels to suppress FAK activity.

Following the first-generation FAK inhibitor drug development including PF-00562271 [10], early drug development of several potent selective second-generation FAK inhibitors has been progressed recently. In the phase 1 dose-escalation study of another second-generation FAK inhibitor BI 853520 , both proteinuria and fatigue were dose-limiting (Grade 3) at the higher doses [11, 12]. In this study, no subject developed any grade of proteinuria; nevertheless, the most common $\mathrm{AE}$ in the general disorders was fatigue reported in $6(67 \%)$ subjects. Fatigue was reported across all dose cohorts with $2(67 \%)$ subjects in the $200 \mathrm{mg}$ dose cohort, 1 (33\%) subject in the $400 \mathrm{mg}$ dose cohort, and $3(100 \%)$ subjects in the $600 \mathrm{mg}$ dose cohort. One subject in the $400 \mathrm{mg}$ dose cohort had an interruption in study drug for 13 days because of moderate fatigue beginning on Day 78. All other reports of fatigue were mild in severity. Regarding safety, increased bilirubin was the most commonly reported laboratory abnormality across all dose cohorts. Most patients had at least one Grade 1 or Grade 2 unconjugated hyperbilirubinemia. Transient increases in total bilirubin were most pronounced at C1D8; however, values for all affected subjects trended toward normal range by the end of the study. In only 1 of the 9 subjects, a dose modification was required for increased bilirubin. The increases observed in blood bilirubin did not appear to be dose related, and were transient and/or sporadic. There was no increase in ALT or AST values associated with the increases in bilirubin. The current hypothesis for occurrence of unconjugated hyperbilirubinemia with VS-6063 is that either the parent compound and/or its metabolite compete with bilirubin for hepatic conjugation and elimination. Of note, both VS-6063 and its metabolites are UGT1A1 inhibitors in vitro. VS-6063 is metabolized in the liver by oxidative metabolism and its metabolite undergoes glucuronide conjugation via uridine diphosphate glucuronosyltransferase (UGT) 1A1, the hepatic enzyme primarily responsible for conjugation of bilirubin [13, 14].
In pharmacokinetics analysis, VS-6063 was rapidly absorbed, with median $T_{\max }$ observed at $2 \mathrm{~h}$ postdose following oral doses of 200-600 mg BID. Plasma VS-6063 exposure $\left(C_{\max }\right.$ and AUC) was less than dose proportional and doses above $400 \mathrm{mg}$ BID did not result in a significant increase in VS-6063 exposure. A similar $C_{\max }$ was observed in the 400 and $600 \mathrm{mg}$ BID dose cohorts on both Days 1 and 15 , and mean AUC values were relatively consistent across the 200-600 mg BID dose range. Clearance appeared to increase with dose on both Days 1 and 15, which is consistent with the less than dose proportional increase in exposure. Exposure at $400 \mathrm{mg}$ BID (RP2D) was comparable to that previously reported in non-Japanese patients [9].

The best overall response in this study was stable disease, reported in 3 of the 9 patients, including 1 patient in each dose cohort. Durable stable disease of approximately 24 weeks was observed in 1 subject with mesothelioma and 1 subject with rectal cancer in the 400 and $600 \mathrm{mg}$ dose cohorts, respectively. No objective radiographic responses were observed with VS-6063, which is similar to findings with the first-generation compound PF-00562271 [10] and another second-generation FAK inhibitors currently in development, GSK2256098 [15] and BI 853520 $[11,12]$. Inhibition of FAK prevents tumor invasion and dissemination rather than reducing tumor bulk. FAK inhibition is not overtly cytotoxic and is expected to be most effective when used in combination with other chemotherapeutic drugs. Recent studies have suggested specific patient populations that may benefit from FAK inhibition. For example, in a signaling network involving extracellular signal-related kinase (ERK), RhoA and FAK are dysregulated in high-grade non-small cell lung cancer (NSCLC) occurring against a background of Ink4a-Arf deficiency [16]. Suppression of RhoA or FAK selectively induced death in mutant Kras/Ink4a-Arf-deficient NSCLC cells, and pharmacologic inhibition of FAK caused regression of high-grade NSCLC in mutant Kras/Cdkn2a-null mice [16]. Based on these data, a study of VS-6063 has been initiated in patients with NSCLC harboring a Kras mutation (ClinicalTrials.gov, NCT01951690). In addition, recent preclinical data revealed that FAK promotes antitumor immune evasion. Specifically, the kinase activity of nuclear-targeted FAK in squamous cell carcinoma (SCC) cells drives exhaustion of CD8 $+\mathrm{T}$ cells and recruitment of regulatory $\mathrm{T}$ cells (Tregs) in the tumor microenvironment by regulating chemokine/cytokine and ligand-receptor networks, including via transcription of $\mathrm{Ccl} 5$, which is crucial. A small molecule FAK kinase inhibitor, VS-4718, which is currently in clinical development, also drives depletion of Tregs and promotes a CD8 + T cell-mediated anti-tumor response. Therefore, FAK inhibitors may trigger immune-mediated tumor regression, providing previously 
unrecognized therapeutic opportunities [17]. These findings could be a strong rationale for combination strategy with FAK inhibitors and immune checkpoint inhibitors including anti-PD-1 and anti-PD-L1 antibodies.

In summary, VS-6063 has an acceptable safety profile with Grade 1-2 adverse events that are easily managed and reversible, even with continued dosing, in Japanese subjects with advanced solid tumor malignancies. PK analyses confirmed that the exposure at the RP2D of $400 \mathrm{mg}$ BID was comparable with that previously reported in non-Japanese subjects. Data from this study support VS-6063 administration to Japanese subjects with advanced solid tumor malignancies in investigational trials. The favorable safety profile of VS-6063 creates the opportunity to combine this drug with other agents or to test it as monotherapy in the adjuvant setting. For use as monotherapy, it will be important to identify suitable candidate populations [18-21].

Acknowledgments The authors would like to thank Archie W. Thurston, Jr, PhD (Seventh Wave Laboratories LLC, Chesterfield, MO, USA) for PK analysis, Scott Murphy (Veristat, LLC, Holliston, MA, USA) for statistical analysis. Research funding was provided by Verastem, Inc. (Needham, MA, USA).

\section{Compliance with ethical standards}

Conflict of interest Joanna Horobin, Mitchell Keegan, Mahesh Padval and Ajit Chavan are employees of Verastem, Inc. and hold stock and stock options of Verastem, Inc. Lou Vaickus is a consultant to Verastem Inc. and holds no stock or stock options. The remaining authors have no potential conflicts of interest to report.

Ethical standards The study was performed in accordance with the ethical standards laid down in the 1964 Declaration of Helsinki and its later amendments. This study was also performed in accordance with Good Clinical Practices (GCP), including the archiving of essential documents. The study was approved by the institutional review board of study site, and all the patients provided informed consent prior to their inclusion in the study.

Open Access This article is distributed under the terms of the Creative Commons Attribution 4.0 International License (http://creativecommons.org/licenses/by/4.0/), which permits unrestricted use, distribution, and reproduction in any medium, provided you give appropriate credit to the original author(s) and the source, provide a link to the Creative Commons license, and indicate if changes were made.

\section{References}

1. Siesser PM, Hanks SK (2006) The signaling and biological implications of FAK overexpression in cancer. Clin Cancer Res 12(11 Pt 1):3233-3237

2. Schwock J, Dhani N, Hedley DW (2010) Targeting focal adhesion kinase signaling in tumor growth and metastasis. Expert Opin Ther Targets 14:77-94

3. Schultze A, Fiedler W (2010) Therapeutic potential and limitations of new FAK inhibitors in the treatment of cancer. Expert Opin Investig Drugs 19:777-788
4. Schlaepfer DD, Mitra SK, Ilic D (2004) Control of motile and invasive cell phenotypes by focal adhesion kinase. Biochim Biophys Acta 1692:77-102

5. Hao H, Naomoto Y, Bao X et al (2009) Focal adhesion kinase as potential target for cancer therapy (Review). Oncol Rep 22:973-979

6. Tilghman RW, Parsons JT (2008) Focal adhesion kinase as a regulator of cell tension in the progression of cancer. Semin Cancer Biol 18:45-52

7. Clarke MF, Dick JE, Dirks PB et al (2006) Cancer stem cellsperspectives on current status and future directions: AACR workshop on cancer stem cells. Cancer Res 66(19):9339-9344

8. Shibue T, Brooks MW, Fatih Inan M et al (2012) The outgrowth of micrometastases is enabled by the formation of filopodiumlike protrusions. Cancer Discov 2(8):706-721

9. Jones SF, Siu LL, Shapiro GI et al (2015) A phase I study of VS-6063, a second-generation focal adhesion kinase inhibitor, in patients with advanced solid tumors. Invest New Drugs 33(5):1100-1107

10. Infante JR, Camidge DR, Mileshkin LR et al (2012) Safety, pharmacokinetic, and pharmacodynamic phase I dose-escalation trial of PF-00562271, an inhibitor of focal adhesion kinase, in advanced solid tumors. J Clin Oncol 30:1527-1533

11. Zer A, Verheijen RB, De Vos F et al (2015) A phase I study of BI 853520 , a potent and selective inhibitor of focal adhesion kinase (FAK), in patients with advanced or metastatic solid tumors. J Clin Oncol 33 (suppl; abstr 2541)

12. Doi T, Lin CC, Ohtsu A et al (2014) A phase I dose-finding study of BI 853520, a potent and selective inhibitor of focal adhesion kinase (FAK), in Japanese and Taiwanese patients with advanced or metastatic solid tumors. Eur J Cancer 50 (suppl 6; abstr 213)

13. Jemnitz K, Lengyel G, Vereczkey L (2002) In vitro induction of bilirubin conjugation in primary rat hepatocyte culture. Biochem Biophys Res Commun 291:29-33

14. Zucker SD, Qin X, Rouster SD et al (2001) Mechanism of indinavir-induced hyperbilirubinemia. Proc Natl Acad Sci USA 98:12671-12676

15. Soria JC, Gan HK, Arkenau HT et al (2012) Phase I clinical and pharmacologic study of the focal adhesion kinase (FAK) inhibitor GSK2256098 in pts with advanced solid tumors. J Clin Oncol 30 (Suppl) Abst. 3000

16. Konstantinidou G, Ramadori G, Torti F et al (2013) RHOA-FAK is a required signaling axis for the maintenance of KRAS-driven lung adenocarcinomas. Cancer Discov 3:444-457

17. Serrels A, Lund T, Serrels B et al (2015) Nuclear FAK controls chemokine transcription, tregs, and evasion of anti-tumor immunity. Cell 163:160-173

18. Poulikakos PI, Xiao GH, Gallagher R et al (2006) Re-expression of the tumor suppressor NF2/merlin inhibits invasiveness in mesothelioma cells and negatively regulates FAK. Oncogene 25:5960-5968

19. Thurneysen C, Opitz I, Kurtz S et al (2009) Functional inactivation of NF2/merlin in human mesothelioma. Lung Cancer 64:140-147

20. Shapiro IM, Kolev VN, Vidal CM et al (2014) Merlin deficiency predicts FAK inhibitor sensitivity: a synthetic lethal relationship. Sci Transl Med 6:237-268

21. Soria JC, Plummer R, Ranson M et al (2012) Loss of the tumor suppressor Merlin as a potential predictive biomarker of clinical activity for the oral, focal adhesion kinase (FAK) inhibitor GSK2256098 in pts with recurrent mesothelioma. Eur J Cancer 48(Suppl 6):188 (Abst. 610) 